\title{
Fluorescence sensing system by Soret-band LED light excitation for estimating relative talaporfin sodium concentration in skin
}

\author{
M. Takahashi MSc*, T. Arai PhD
}

School of Fundamental Science and Technology, Graduate School of Science and Technology, Keio University, 3-14-1, Hiyoshi, Kohoku-ku, Yokohama City, Kanagawa 223-8522, Japan

\author{
KEYWORDS \\ Skin photosensitivity; \\ Fluorescence; \\ Medical diagnosis; \\ Photodynamic \\ therapy; \\ Talaporfin sodium
}

\begin{abstract}
Summary The purpose of this study is to establish a sensing system to estimate relative talaporfin sodium concentration in skin to evaluate the risk of skin photosensitivity after photodynamic therapy (PDT) using percutaneous fluorescence spectroscopy. A prototype fluorescence sensing probe was made using a pair of $5-\mathrm{cm}$-long diffuse tips of plastic optical fibers for excitation light irradiation and fluorescence collection. Talaporfin sodium $(2.5 \mathrm{mg} / \mathrm{kg})$ was intravenously administrated to three pigs, and the talaporfin sodium concentration in plasma was measured. The fluorescence sensing probe was attached to the skin and excited by a LED light with a peak wavelength of $409 \pm 16 \mathrm{~nm}$ to obtain the mean area of the talaporfin sodium fluorescence spectral peak $\left(S_{\text {fluo }}\right)$. The time history of the talaporfin sodium concentration in tissue was estimated using a two-compartment pharmacokinetic model. The time history of $S_{\text {fluo }}$ was described as a composite function of the time history of the measured talaporfin sodium concentration in plasma and that of the estimated concentration in tissue as a double exponential decay function. The relative talaporfin sodium concentration in tissue and the relative contributions of fluorescence from tissue and plasma to $S_{\text {fluo }}$ were estimated by the fluorescence system with the numerical pharmacokinetic model. Results also show that tissue compression equivalent to venous pressure might be effective to suppress the contribution of talaporfin sodium fluorescence in plasma.
\end{abstract}

(c) 2014 Elsevier B.V. All rights reserved.
* Corresponding author. Tel.: +81 45563 1151x42580; fax: +81455661587.

E-mail addresses: mei.60613195@a3.keio.jp, m.takahashi@arai.appi.keio.ac.jp (M. Takahashi), tsunearai@appi.keio.ac.jp (T. Arai).

\section{Introduction}

Skin photosensitivity is a major side effect after photodynamic therapy (PDT), although PDT has been widely performed in cancer therapy, such as gastroenterology, thoracic surgery, dermatology, and neurosurgery [1-3]. Patients are forced to remain hospitalized in darkness, e.g., 1 month for photofrin administration in gastric, esophageal, and early stage lung cancer therapy, to avoid skin

1572-1000/@ 2014 Elsevier B.V. All rights reserved. 
photosensitivity [2-5]. Skin photosensitivity exacerbates sunburn due to the photosensitization reaction caused by exposure to light that excites the photosensitizer remaining in the skin after PDT [6]. Skin photosensitivity occurs mainly in the epidermis, which has a depth of $65-130 \mu \mathrm{m}$, due to the penetration depth of the light absorbed by the photosensitizer, such as 400-nm-band or ultraviolet (UV) light [7-9]. Symptoms of skin photosensitivity include pruritus, erythema, edema in the dermis, and blisters $[6,7,10]$, and result from epidermal cell degeneration and dermal inflammatory response $[10,11]$.

PDT using talaporfin sodium, a second-generation photosensitizer, has been approved and utilized in therapy for cancers, such as early stage lung cancer and malignant glioma, in Japan [3,4,12]. In addition, we have proposed application of PDT using talaporfin sodium for myocardial ablation to treat tachyarrhythmia [13,14]. Talaporfin sodium is a water-soluble photosensitizer and exhibits strong absorption at $412 \mathrm{~nm}$ for the Soret-band and $663 \mathrm{~nm}$ for the Q-band [15]. After administration, talaporfin sodium is rapidly metabolized by the liver, with a half-life of $9.0 \pm 0.8 \mathrm{~h}$ in the fast phase of pharmacokinetics in humans [16]. In the currently approved PDT protocol using talaporfin sodium, hospitalization in darkness for a period of 2 weeks is prescribed to avoid skin photosensitivity $[3,4,17]$. However, the metabolism of talaporfin sodium shows wide variety among individuals; therefore, the incidence rate of skin photosensitivity differs between individuals. For example, in a clinical trial of PDT using talaporfin sodium for malignant glioma in Japan, skin photosensitivity disappeared in $55.6 \%$ of patients 4 days after administration, $77.8 \%$ of patients 8 days after administration, and none of the patients exhibited any signs of skin photosensitivity by 16 days after the administration based on the currently approved protocol for judging the risk of skin photosensitivity $[4,17]$. Therefore, it might be possible to shorten the time period for hospitalization in darkness for patients with high metabolic rates.

A reliable methodology to manage the risk of skin photosensitivity would be helpful for shortening the dark hospitalization period. The current protocol for judging the risk of skin photosensitivity is to expose patient's fingers and palm to sunlight for $5 \mathrm{~min}$ to see whether the skin photosensitivity reaction occurs. This protocol is repeated until the photosensitivity reaction is not seen $[3,4,17]$. This methodology is simple and easy to perform; however, it is invasive and has poor reliability.

The aim of this study is to establish a noninvasive optical sensing system capable of estimating relative concentration of talaporfin sodium in skin using fluorescence spectroscopy to evaluate the risk of skin photosensitivity. It is reported that skin photosensitivity does not occur when the concentration of talaporfin sodium in plasma is in the range of $0.6-2.9 \mu \mathrm{g} / \mathrm{ml}$ [18], whereas the talaporfin sodium concentration in skin would be approximately 5.8 times higher than in plasma 7 days after administration [19]. Therefore, the required measurement sensitivity is at least $3.0 \mu \mathrm{g} / \mathrm{ml}$ in plasma to evaluate the risk of skin photosensitivity.

There are several reports that estimate the relative photosensitizer concentration in tissue or optical phantoms. For example, Canpolat et al. and Weersink et al. reported on reflectance spectroscopy using fiber bundles to estimate the absorption coefficient of m-tetrahydroxyphenylchlorin
(mTHPC) in an optical phantom or aluminum phthalocyanine tetrasulfonate $\left(\mathrm{AlPcS}_{4}\right)$ in rabbit skin, respectively $[20,21]$. However, there is no report of a method or device to predict the relative talaporfin sodium concentration in skin with the aim of evaluation of skin photosensitivity risk.

We designed a fluorescence sensing system to estimate the relative talaporfin sodium concentration in skin employing Soret-band LED light excitation and fluorescence spectral measurement via a pair of diffuse tips made of plastic optical fibers. The properties and performance of this fluorescence sensing system were investigated in vitro. Fluorescence spectra from the skin of pigs administered talaporfin sodium and the talaporfin sodium concentration in plasma were then measured in vivo to demonstrate the estimation of relative talaporfin sodium concentration in skin.

\section{Materials and methods}

\section{Design of the fluorescence sensing system}

Because the fluorescence measurement would be performed simply before and after PDT, cutaneous measurement on a part of the body not covered with clothing on a daily basis, such as the upper arms, would be preferable. However, the microscopic appearance of the skin surface is nonhomogeneous because of the distribution of sweat pores, lentigo, and hair [8]; therefore, we employed a large sampling area on the skin using two diffuse tips made of 5 -cm-long plastic optical fibers in the fluorescence sensing probe to average the anatomical differences in regions of the skin.

A length of each plastic optical fiber $(1 \mathrm{~mm} \Phi$, PGU FB1000, Toray Industries, Japan) $5 \mathrm{~cm}$ from the distal end was clad-polished using sandpapers \#280 and \#1000 while checking the light radiation from the diffuse tip to obtain a uniform light distribution. A schematic diagram of the fluorescence sensing probe is shown in Fig. 1.

The diffuse tips and fibers were aligned next to each other to irradiate the excitation light and collect the fluorescence from talaporfin sodium. Urethane gel with a thickness of $1.0 \pm 0.1 \mathrm{~mm}$ was employed as a buffer material to attach the fluorescence sensing probe to the skin. The urethane gel and the diffuse tips were adhered together by double-sided adhesive tape. A thermocouple (TH-8162-2, ThreeHigh Co., Ltd., Japan) was also placed on the urethane gel to measure the temperature of the skin surface and assess whether the blood flow of the skin surface was maintained during application of the fluorescence sensing probe. Black masking tape was placed over the diffuse tips and thermocouples to block environmental light.

Blue LED light with a center wavelength of $409 \mathrm{~nm}$ and a full width at half maximum (FWHM) of $16 \mathrm{~nm}$ (LLS405, Ocean Optics, Inc., Dunedin, FL) was used to excite talaporfin sodium in the Soret-band, in which the molar absorption coefficient of talaporfin sodium is comparatively high at $1.8 \times 10^{5} \mathrm{M}^{-1} \mathrm{~cm}^{-1}[7-9,16]$. The penetration depth of this wavelength in skin is reported to be $90-300 \mu \mathrm{m}$, so the sampling region of the fluorescence sensing system is expected to be the epidermis and dermis [7-9,22-25]. The distribution of talaporfin sodium in skin might be homogeneous because it is reported that talaporfin sodium 


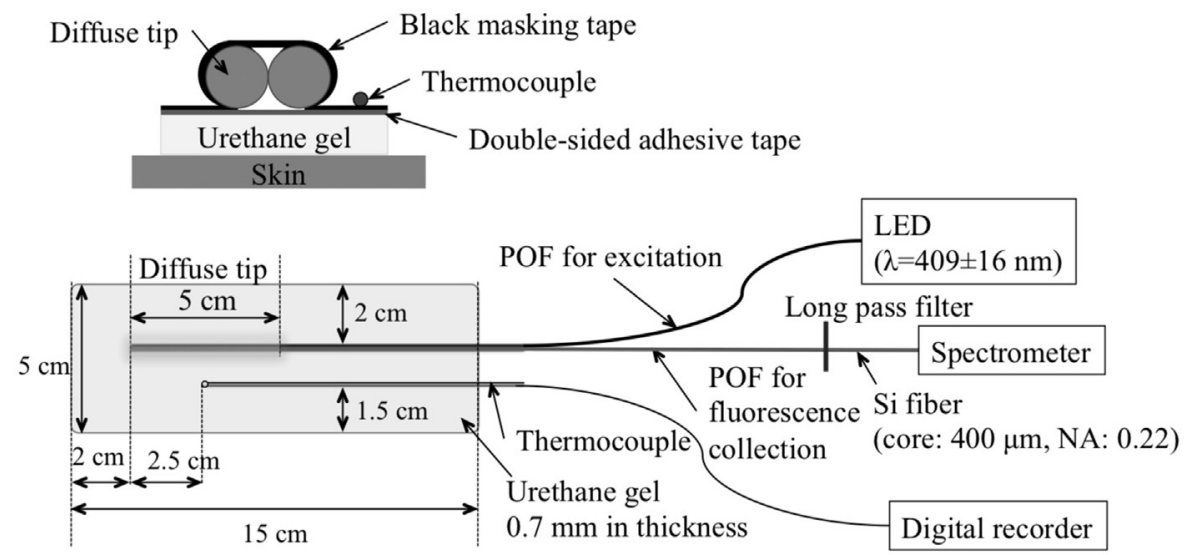

Figure 1 Schematic diagram of the epidermal photosensitivity sensing system. A pair of diffused tips made of plastic optical fibers (POF) and a thermocouple were embedded in urethane gel to irradiate the excitation light and collect the fluorescence. Upper image: Side view. Lower image: Front view.

was uniformly distributed in normal mouse ear observed by in vivo confocal imaging in the optical section of $600 \mu \mathrm{m} \times 600 \mu \mathrm{m}$ with thickness of $6 \mu \mathrm{m}$ [26]. The fluorescence collected via the diffuse tip was introduced into measurement optics, which included a 600-nm long-pass filter (FEL0600, Thorlabs Japan, Japan) to eliminate the excitation light, environmental light, and skin tissue autofluorescence. The spectra of the excitation light and the long-pass filter transmittance are shown in Fig. 2. Spectral measurement was performed with a spectrometer (FL4000, Ocean Optics, Inc., Dunedin, FL) in the wavelength range of $400-800 \mathrm{~nm}$.

Performance of the fluorescence sensing system in vitro The light transmission rate for the $5-\mathrm{cm}$-long diffuse tip and a 1.7-m-long plastic optical fiber were measured using an integrating sphere (3P-GPS-040-SF, Labsphere Inc., North Sutton, NH) and a power meter (PowerMax-USB UV/VIS, Coherent Japan, Inc., Japan). Light irradiation distributions from both diffuse tips for excitation light irradiation and fluorescence collection in the fluorescence sensing probe were measured. The input power of the blue LED light

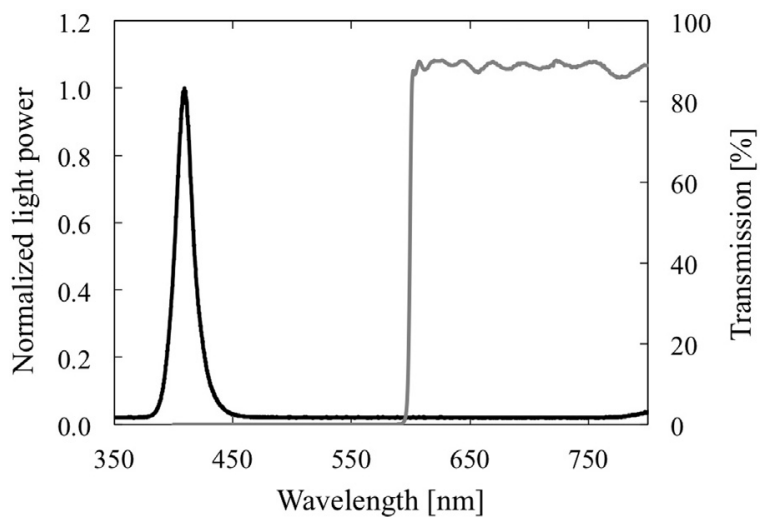

Figure 2 Spectra of excitation light and long-pass filter (LPF) transmission. The power of the excitation light was normalized at the peak wavelength of $409 \pm 16 \mathrm{~nm}$. Black line: Excitation light. Gray line: LPF transmission. for both diffuse tips was $11 \mathrm{~mW}$. The emitted light power distribution from the diffuse tip in the major axis of the plastic optical fiber was measured via a silica optical fiber with a core diameter of $200 \mu \mathrm{m}$ and a numerical aperture (NA) of 0.53 (WF200/220HT53, CeramOptec Industries, Inc., East Longmeadow, MA) using another power meter (OP-2 VIS, Coherent Japan, Inc., Japan). The fiber position was controlled using a motorized stage (SGSP20-85, Sigmakoki Co., Ltd., Japan). The light distribution along the minor axis was measured using the same set up.

\section{Observation of pig skin structure and application of the fluorescence sensing system in vivo}

\section{Animal preparation and observation of skin structure}

The animal experiment was conducted in compliance with the Declaration of Helsinki, and conducted with the approval of the ethics committee of IVTeC Co., Ltd., where the in vivo experiment was conducted, and the Animal Care Committee at Keio University, Japan. Three Large White breed (LW), female pigs (mean body weight, $27.7 \pm 1.1 \mathrm{~kg}$ ) were anesthetized with $3 \%$ isoflurane and pure oxygen at $1.5-2.0 \mathrm{l} / \mathrm{ml}$. Deep anesthesia was maintained throughout the procedure. Standard limb-lead electrocardiogram, heart rate, blood pressure, oxygen saturation by pulse oximetry, and body temperature were monitored continuously throughout the procedures.

To confirm the tissue structure in the sampling area of the skin photosensitivity sensing system, histological specimens were microscopically observed. Back skin tissue specimens $\left(7 \mathrm{~cm}^{2}\right)$ from the pigs were extracted, fixed with $10 \%$ formalin, and embedded in paraffin. The paraffin-embedded sections were then microsectioned at a thickness of $6 \mu \mathrm{m}$ and stained with hematoxylin and eosin (HE). These specimens were observed using a microscope (FSX100, Olympus Co., Japan) at $40 \times$ magnification.

\section{Measurement of cutaneous fluorescence}

Talaporfin sodium $(2.5 \mathrm{mg} / \mathrm{kg})$ was administered via the right cervical vein. Blood $(4 \mathrm{ml})$ was drawn from the left cervical vein to measure the talaporfin sodium concentration in 
(a)

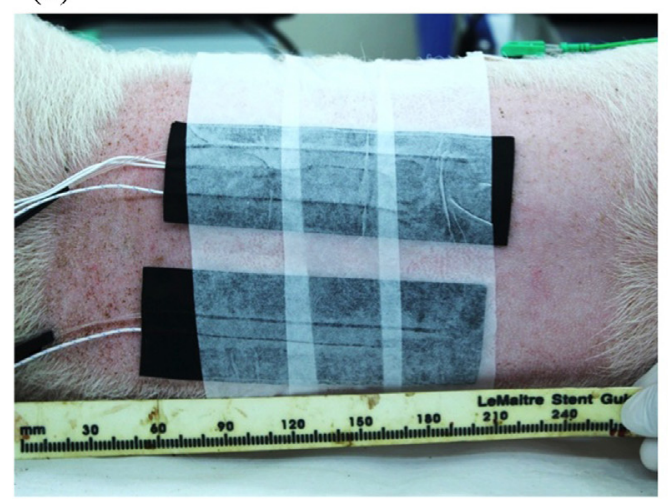

(b)

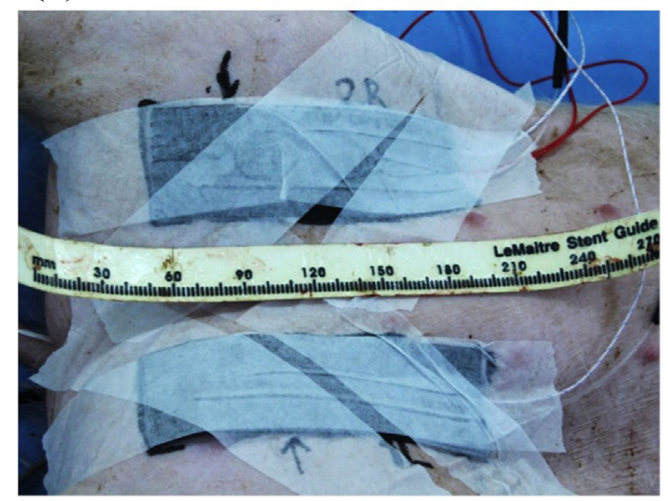

Figure 3 Image showing application of the epidermal photosensitivity sensing probe on the back (a) and lower limb (b) of a pig. After the body hair was shaved, the epidermal photosensitivity sensing probes were attached using surgical tape.

plasma before the administration as a control, and every $5 \mathrm{~min}$ after the administration up to $90 \mathrm{~min}$. The collected blood was centrifuged at $3000 \mathrm{rpm}$ for $10 \mathrm{~min}$. The plasma was collected from the supernatant to measure the absorption spectrum of talaporfin sodium, its plasma concentration was calculated using a spectrophotometer (UV3600, Shimadzu Co., Japan) in the wavelength range of $350-750 \mathrm{~nm}$. The area of the absorption peak of talaporfin sodium in the wavelength range of $624-691 \mathrm{~nm}$ was calculated, and the mean concentration of talaporfin sodium in plasma was estimated based on a calibration curve, which was determined in a preliminary experiment using high-performance liquid chromatography.

The fluorescence sensing probe was applied to the skin of the back and both lower limbs using surgical tape, as shown in Fig. 3. The blue LED light of $11 \mathrm{~mW}$ was inputted into the diffuse tip for fluorescence collection in the fluorescence sensing probe, therefore the irradiance was $4.3 \mathrm{~mW} / \mathrm{cm}^{2}$. Compression of $200 \mathrm{~g} / \mathrm{cm}^{2}$, which is greater than venous blood pressure, was applied on the right lower limb to investigate the effect of pressure on fluorescence spectral measurements. Each spectral measurement was conducted for 10 s using the same optics as described in section "Design of the fluorescence sensing system', with an integration time of $2 \mathrm{~s}$. These measurements were performed before talaporfin sodium administration as a control and every $5 \mathrm{~min}$ after the administration up to $90 \mathrm{~min}(\mathrm{~N}=3)$.

The temperature of the skin surface was continuously measured using the thermocouple embedded in the fluorescence sensing probe and recorded by a digital recorder (DL750, Yokogawa Electric Corporation, Japan).

After all of the spectral measurements described above, the measured skin regions were observed with the naked eye and relative tissue oxygen saturation was measured using a tissue oxygenation spectral analyzer (C9183, Hamamatsu Photonics, Japan) to investigate the effect of applying the fluorescence sensing system on the skin.

\section{Fluorescence spectral analysis}

The fluorescence spectra measured using the fluorescence sensing system include the fluorescence from talaporfin sodium in skin tissue and tissue autofluorescence. Because the tissue autofluorescence must be suppressed in order to accurately measure the fluorescence from the talaporfin sodium distributed in epidermis, the 600 -nm long-pass filter was employed in the measurement set up as described above. The talaporfin sodium fluorescence is also affected by variations in the tissue pigmentation and the structure of the measured tissue region. Therefore, we calculated the mean area of the fluorescence spectra of talaporfin sodium in the wavelength range of $660-690 \mathrm{~nm}$ using a baseline method. The baseline $f(\lambda)$ was selected as a straight line passing through the points of the fluorescence spectra at wavelengths of 660 and $690 \mathrm{~nm}$, described as follows:

$f(\lambda)=\left(\frac{\text { fluo }_{690}-\text { fluo }_{660}}{690_{\mathrm{nm}}-660_{\mathrm{nm}}}\right)\left(\lambda-660_{\mathrm{nm}}\right)+$ fluo $_{660}$,

where fluo $_{690}$ and $\mathrm{fluo}_{660}$ are the measured intensities of the fluorescence at wavelengths of 690 and $660 \mathrm{~nm}$, respectively.

The mean area of the fluorescence spectra of talaporfin sodium in the wavelength range of $660-690 \mathrm{~nm}$ was calculated using the following formula for fluorescence peak area $\left(S_{\text {fluo }}\right)$ :

$S_{\text {fluo }}=\int_{660 \mathrm{~nm}}^{690 \mathrm{~nm}}\left(\right.$ fluo $\left._{\lambda}-f(\lambda)\right) d \lambda$.

\section{Pharmacokinetic analysis}

Pharmacokinetic analysis was performed to predict the time history of talaporfin sodium concentration in tissue based on the measured talaporfin sodium concentration in plasma with the aim of interpret the measured $S_{\text {fluo }}$ to distinguish the origin of the measured fluorescence.

Since the pharmacokinetics of a drug are generally complex, mathematical models have been utilized to aid in understanding them $[26,21]$. A two-compartment model, one of the major pharmacokinetic models, was employed to analyze talaporfin sodium metabolism in pig skin. In this model, time decays of the talaporfin sodium concentration in both plasma and tissue were described using a double exponential function, as the time history of 

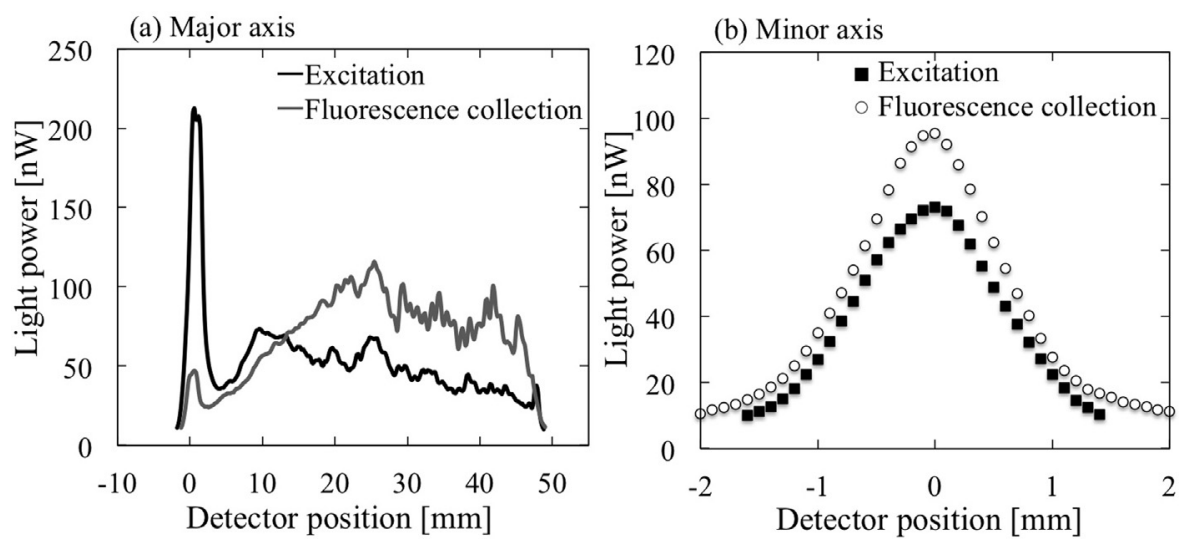

Figure 4 Light distribution of the pair of diffuse tips of plastic optical fibers (POF) for both excitation and fluorescence collection in the epidermal photosensitivity probe. (a) The major axis direction. The detector position of 0 represents the distal end of the diffuse tip. Black line: POF for excitation, Gray line: POF for fluorescence collection. (b) The minor axis direction. The detector position of 0 represents the center of the diffuse tip. Squares: POF for excitation, Circles: POF for fluorescence collection.

talaporfin sodium concentration in plasma $P(t)$ and in tissue $Q(t)$, shown as follows [21]:

$P(t)=A \exp (-\alpha t)+B \exp (\beta t)$

$Q(t)=\frac{D(A \beta+B \alpha)}{V_{1}(A+B)}(-\exp (-\alpha t)+\exp (-\beta t))$

where $A, B, \alpha$, and $\beta$ are constants, $\alpha>\beta, D$ is the talaporfin sodium dosage, and $V_{1}$ is the volume distribution of talaporfin sodium in blood $[21,27]$. The measured talaporfin sodium in plasma was fitted to a double exponential curve using a least-squares method to estimate the time history of talaporfin sodium in tissue $Q(t)$.

Since the measured fluorescence of talaporfin sodium excited by Soret-band LED light using the fluorescence sensing system might originate from talaporfin sodium distributed in both tissue and plasma, the time history of $S_{\text {fluo }}$ can likely be described by a composite function of $P(t)$ and $Q(t)$ in a double exponential decay function as follows:

$S_{\text {fluo }}=a P(t)+b Q(t)$,

where $a$ and $b$ are constants. The measured time histories of $S_{\text {fluo }}$ were fitted according to (5), and the constants $a$ and $b$ were derived. The ratio of $a$ to $b, a / b$, which reflects the contribution of talaporfin sodium fluorescence from plasma relative to that from tissue, was calculated.

\section{Results}

\section{Performance of the fluorescence sensing system in vitro}

The light transmission rate of the diffuse tip used in the fluorescence sensing probe was $61.4 \pm 1.8 \%$. Typical light emission distributions from both diffuse tips for excitation light irradiation and fluorescence collection embedded in the fluorescence sensing system are shown in Fig. 4.

\section{Observation of skin structure}

A typical HE-stained specimen of the back skin of a pig observed at $40 \times$ magnification is shown in Fig. 5. The measured thickness of the epidermis was $90.6 \pm 21.3 \mu \mathrm{m}$, which was within the range of reported values [7-9]. Arterioles and venules were found at a depth of $256.6 \pm 62.5 \mu \mathrm{m}$. The pig skin structure was non-homogeneous, and the location of capillaries, arterioles, and venules varied between specimens.

\section{Application of the fluorescence sensing system in vivo and pharmacokinetic analysis}

A typical measured fluorescence spectrum of the back skin of a pig is shown in Fig. 6. The peak fluorescence wavelength of talaporfin sodium was $667 \mathrm{~nm}$ [16]. There was no photobleaching of fluorescence during each $10 \mathrm{~s}$ measurement, and the skin surface temperature remained within $34.2 \pm 0.6^{\circ} \mathrm{C}$ throughout the measurement. HE-stained specimens did not show any evidence of inflammation or blood vessel occlusion. Moreover, the relative tissue oxygen saturation was $9.0 \pm 1.9$ for the skin to which the fluorescence sensing probe was applied and $9.8 \pm 3.4$ for control skin. The peak intensity of talaporfin sodium fluorescence spectra at $667 \mathrm{~nm}$ gradually decreased with time after the administration, which might reflect the egestion of talaporfin sodium.

The time history of the measured talaporfin sodium concentration in plasma and the estimated talaporfin sodium concentration in tissue based on the two-compartment pharmacokinetic model are shown in Fig. 7. The minimum concentration of talaporfin sodium in plasma was $2.5 \pm 0.2 \mu \mathrm{g} / \mathrm{ml} 90 \mathrm{~min}$ after administration, which is equivalent to the required sensitivity to evaluate the risk of skin photosensitivity.

The typical time history of $S_{\text {fluo }}$, measured talaporfin sodium concentration in plasma, and estimated talaporfin sodium concentration in tissue were normalized at the maximum of each dataset and are plotted in Fig. 8. The absolute values of each data are indicated in Fig. 8 by the right-side axes. The time history of $S_{\text {fluo }}$ showed an exponential decay 


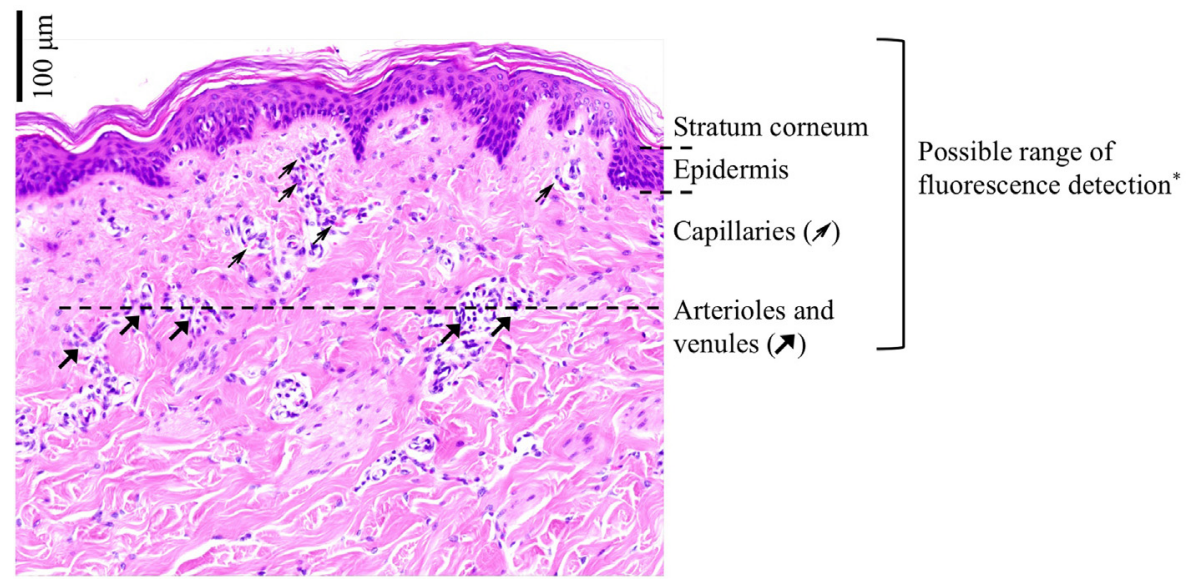

Figure 5 Approximate tissue structure of the back skin of pigs. HE-stained specimens were observed at 40x magnification. *The method of prediction for possible range of fluorescence detection is described in section "Discussion".

in the same manner as both the measured concentration in plasma and estimated concentration in tissue. The calculated $a / b$ based on composite function fitting of $S_{\text {fluo }}(t)$ using (5) is shown in Fig. 9. The calculated $a / b$ was in the range of $0.25-0.76$ in most cases. Extremely high values of $a / b$ were obtained in the back skin of Pig 3. Compression of limb skin tended to decrease $a / b$.

\section{Discussion}

The measured time history of $S_{\text {fluo }}$ using a fluorescence sensing system with Soret-band LED light excitation revealed the time history characteristics of the talaporfin sodium concentration in both plasma and tissue. The relative contribution of the talaporfin sodium concentration in plasma and tissue $(a / b$; described by $(5))$ is shown in Fig. 9. Excluding some exceptionally high values from the back skin of Pig 3 and limb skin of Pig 1 without compression, the mean $a / b$ value was $0.61 \pm 0.17$, representing a plasma to tissue ratio of $3: 5$. This ratio might translate into the sampling depth ratio, assuming a uniform fluorescence intensity distribution in the depth direction for simplification. Assuming that the fluorescence sampling area comprised the

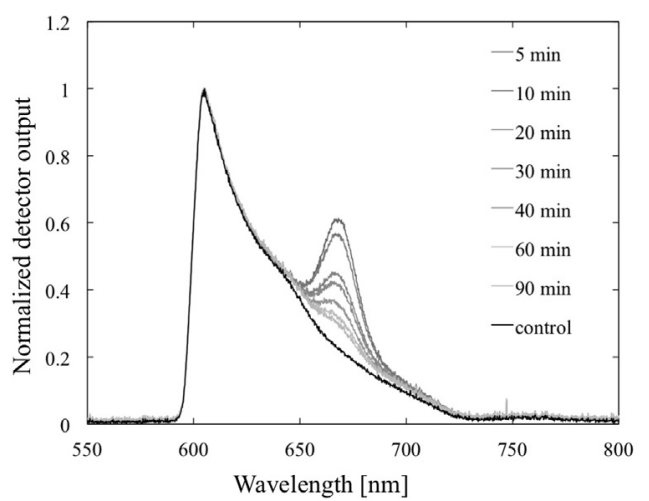

Figure 6 Collected fluorescence spectra measured in the back skin of pigs from before (control) to $90 \mathrm{~min}$ after talaporfin sodium administration. The spectra were normalized using the maximum detector output at $605 \mathrm{~nm}$. epidermis and dermis, including capillaries, arterioles, and venules, the fluorescence from the epidermis would correspond to that from tissue and the fluorescence from the dermis would correspond to that from plasma. Thus, the depth of the dermis can be calculated as approximately $54.4 \pm 12.8 \mu \mathrm{m}$, while the thickness of the epidermis is $90.6 \pm 21.3 \mu \mathrm{m}$ based on microscopic observation of HEstained specimens. The sampling depth of the fluorescence can be estimated as $145.0 \pm 34.1 \mu \mathrm{m}$, which is equivalent to the reported penetration depth of the Soret-band excitation light in skin: $90-300 \mu \mathrm{m}$ [22-25]. Since the estimated fluorescence sampling depth was in the range of reported values, it was confirmed that the measured $S_{\text {fluo }}$ based on the fluorescence measurement using the fluorescence sensing probe reflects the relative talaporfin sodium concentration in skin tissue and plasma. Moreover, the relative remaining talaporfin sodium concentration in skin can be estimated by this measurement and talaporfin sodium pharmacokinetics in plasma. However, $a / b$ showed some exceptionally large values, greater than 2.0, in the back skin of Pig 3. This might be because large blood vessels in the area measured using the fluorescence sensing system increased the contribution

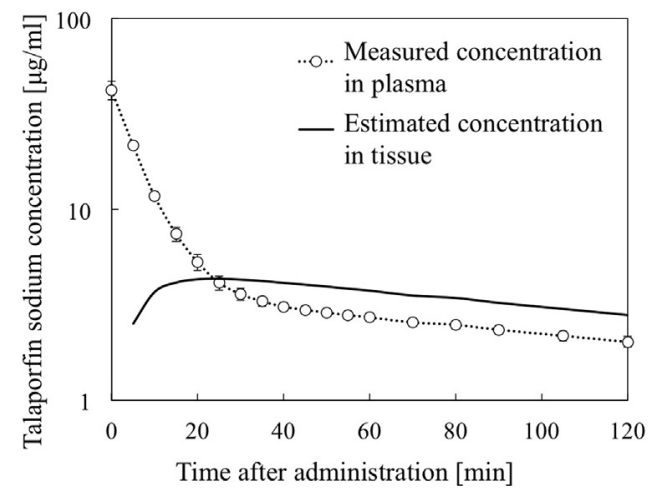

Figure 7 Time history of the measured concentration of talaporfin sodium in plasma and estimated concentration of talaporfin sodium in tissue. The plot indicates the mean measured concentration of talaporfin sodium in plasma at each time point in three pigs. 


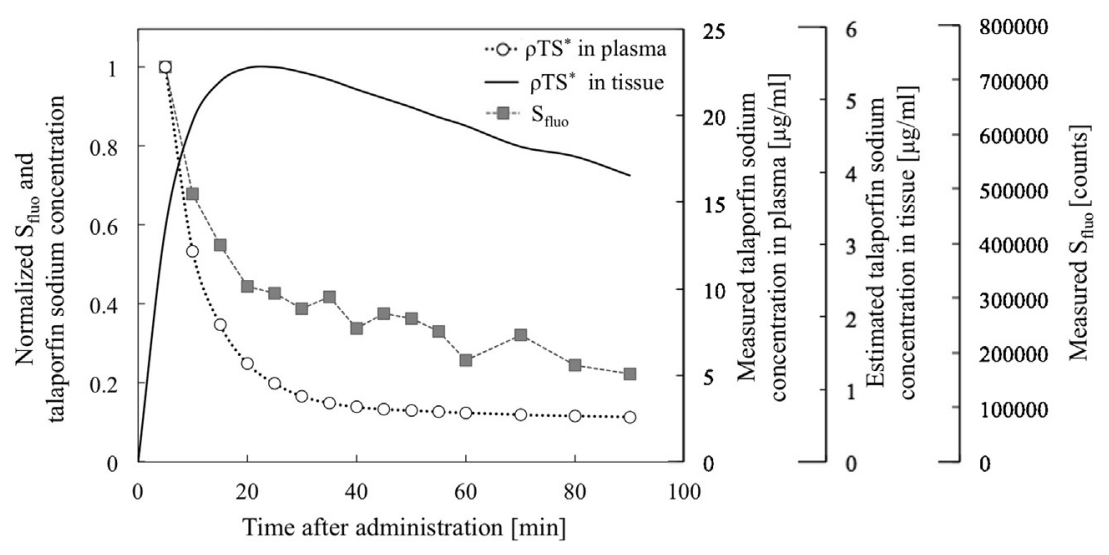

Figure 8 Typical time history of $S_{\text {fluo, }}$, measured talaporfin sodium concentration in plasma, and estimated talaporfin sodium concentration in tissue. The plots of measured talaporfin sodium concentration in plasma, and estimated talaporfin sodium concentration in tissue were reproduction - described from Fig. 7. Plots were normalized using the maximum of each dataset to describe the time history change using left-side axis. The absolute values of each plots were described by the right-side axis. White circles: Measured talaporfin sodium concentration in plasma. Black solid line: Estimated talaporfin sodium concentration in tissue. Gray rectangles: Sfluo measured in limb skin of Pig 2 without compression.

of the talaporfin sodium in plasma. For these cases, compression greater than the venous blood pressure might be useful to reduce the contribution of fluorescence of talaporfin sodium in plasma to $S_{\text {fluo }}$ as indicated by the results shown in Fig. 9. It might be because of the compression might exclude the blood circulating in the sampling area of the fluorescence probe.

The fluorescence measurement was performed while the talaporfin sodium concentration in plasma was $2.5 \pm 0.2 \mu \mathrm{g} / \mathrm{ml}$, which is equivalent to the reported talaporfin sodium concentration in human plasma $336 \mathrm{~h}$ after administration, when almost all patients no longer exhibit skin photosensitivity $[18,28]$. More specifically, we performed the fluorescence measurement until $90 \mathrm{~min}$ after the administration in pigs; however, this measurement was equivalent to that in humans $336 \mathrm{~h}$ after administration. This is because the pharmacokinetics of talaporfin sodium in pigs are 30 times as fast as in humans [18]. Therefore, the

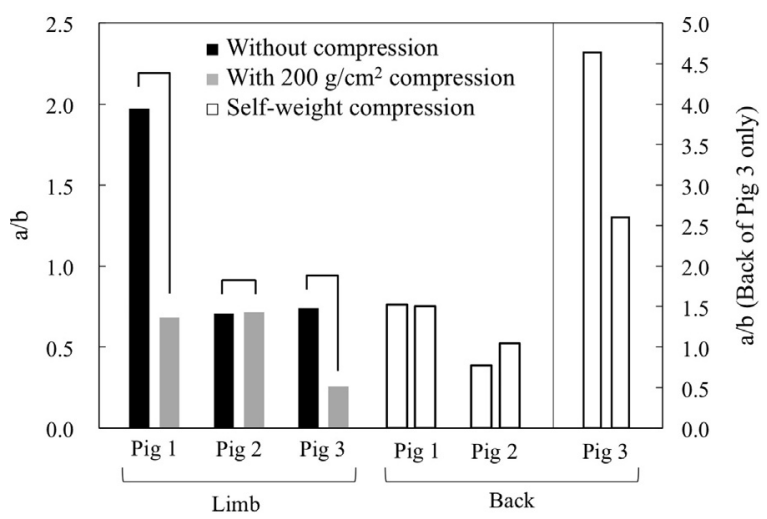

Figure 9 Calculated ratio $a / b$ based on the composite function fitting of $S_{\text {fluo }}(t)$ using (5). The right horizontal axis is for only plots of data from the back of Pig 3. Black: Without compression. Gray: Compression applied. White: Self-weight compression. fluorescence sensing system has adequate sensitivity to detect the talaporfin sodium fluorescence remaining in skin after PDT to evaluate the risk of skin photosensitivity.

Calculated $S_{\text {fluo }}$ based on the fluorescence measurement showed variations in animals, although the measured time decay of talaporfin sodium concentration in plasma was almost the same for each animal. The variation in $S_{\text {fluo }}$ might be because of the difference in the remaining talaporfin sodium concentration in skin among individuals due to the specificity of each animal's metabolism. Skin is a peripheral tissue, and pigs metabolize talaporfin sodium very fast, so that the variety in metabolism among individuals in skin might be emphasized [17]. Moreover, $S_{\text {fluo }}$ might be affected by the non-homogeneity of the skin structure and the variety of optical properties such as skin color. The $5-\mathrm{cm}$ long diffuse tip of the fluorescence sensing probe might be effective to average the non-homogeneity of the skin structure, and the normalization of $S_{\text {fluo }}$ just after the talaporfin sodium administration might be useful to compensate for the variation in optical properties to analyze the individual metabolism of talaporfin sodium in skin.

The safety of the fluorescence sensing system must be confirmed by not only histological observation but also the damage threshold of skin due to PDT. In the case of the fluorescence sensing system, the radiant exposure to the skin in one measurement was $5.8 \mathrm{~mJ} / \mathrm{cm}^{2}$. On the other hand, it is reported that the damage threshold of skin due to PDT was approximately $2 \mathrm{~J} / \mathrm{cm}^{2}$ [28]. In addition, photobleaching during the measurements using the fluorescence sensing system was not observed, leading us to believe that the photosensitive reaction of PDT scarcely occurs due to the application of the fluorescence sensing system [29]. Therefore, fluorescence measurement using the developed fluorescence sensing system would be safe.

The advantage of the fluorescence sensing system lies in the use of plastic optical fibers for the diffuse tip. Plastic optical fibers are generally more flexible compared to silica fibers, which makes using them in clinical applications easier. Moreover, the plastic fibers are inexpensive. 
With these characteristics and the ability to estimate the relative talaporfin sodium concentration, the fluorescence sensing system would be useful to evaluate the risk of skin photosensitivity after PDT using talaporfin sodium.

Although the efficacy and safety of the fluorescence sensing system were confirmed, there are several limitations. First, the absolute concentration of talaporfin sodium in skin cannot be estimated by fluorescence measurement using the fluorescence sensing system. An additional calibration measurement would be needed to analyze the talaporfin sodium concentration in the skin quantitatively. Second, although the localized pigmentation variety might be successfully compensated by the long sampling length of $5 \mathrm{~cm}$ for the diffuse tip and the normalization of $S_{\text {fluo }}$, the fluorescence might not be measured if the absorption coefficient is extremely high $[24,30]$.

\section{Conclusion}

We demonstrated that relative talaporfin sodium concentration distributed in skin was successfully detected using the fluorescence sensing system employing Soret-band LED light excitation in pig model. Since we estimated that over $60 \%$ portion of the measured fluorescence comes from epidermis and the detectivity of the estimated relative talaporfin sodium concentration was equivalent to sufficient detectivity to clinical application, we think our system may be applicable to evaluate skin photosensitivity risk.

\section{Acknowledgement}

This work was supported in part by the Japan Science and Technology Agency Adaptable \& Seamless Technology Transfer Program through Target-driven R\&D (A-STEP); Contract grant number: AS2415004P; and the Japan Society for the Promotion of Science (Research Fellowships for Young Scientists); Contract grant number: 5130. The authors sincerely thank Mr. Kawakami, Mr. Yajima, Mr. Takenoya, and Mr. Matsuzaki for their support with the in vivo experiments.

\section{References}

[1] Ackroyd R, Kelty C, Brown N, Reed M. The history of photodetection and photodynamic therapy. Photochem Photobiol 2001;74:656-69.

[2] Usuda J, Kato H, Okunaka T, Furukawa K, Tsutsui H, Yamada K, et al. Photodynamic therapy (PDT) for lung cancers. J Thorac Oncol 2006;1:489-93.

[3] Kato H, Furukawa K, Sato M, Okunaka T, Kusunoki Y, Kawahara $M$, et al. Phase II clinical study of photodynamic therapy using mono-l-aspartyl chlorin e6 and diode laser for early superficial squamous cell carcinoma of the lung. Lung Cancer 2003;42:103-11.

[4] Muragaki Y, Akimoto J, Maruyama T, Iseki H, Ikuta S, Nitta $M$, et al. Phase II clinical study on intraoperative photodynamic therapy with talaporfin sodium and semiconductor laser in patients with malignant brain tumors. J Neurosurg 2013;119:845-52.

[5] Dougherty TJ, Cooper MT, Mang TS. Cutaneous phototoxic occurrences in patients receiving Photofrin ${ }^{\circledR}$. Lasers Surg Med 1990;10:485-8.

[6] Gould JW, Mercurio MG, Elmets CA. Cutaneous photosensitivity diseases induced by exogenous agents. J Am Acad Dermatol 1995;33:551-73.

[7] Goff BA, Bachor R, Kollias N, Hasan T. Effects of photodynamic therapy with topical application of 5 -aminolevulinic acid on normal skin of hairless guinea pigs. J Photochem Photobiol B 1992;15:239-51.

[8] Montagna W, Kligman AM, Carlisle KS. Epidermis. Atlas Normal Hum Skin 1992;1:8-24.

[9] Meyer W, Kacza J, Zschemisch NH, Godynicki S, Seeger J. Observations on the actual structural conditions in the stratum superficiale dermidis of porcine ear skin, with special reference to its use as model for human skin. Ann Anat 2007;189: $143-56$.

[10] Dillon J, Kennedy JC, Pottier RH, Roberts JE. In vitro and in vivo protection against phototoxic side effects of photodynamic therapy by radioprotective agents WR-2721 and WR-77913. Photochem Photobiol 1988;48:235-8.

[11] Sigdestad CP, Fingar VH, Wieman TJ, Lindberg RD. Chemical modification of normal tissue damage induced by photodynamic therapy. Br J Cancer Suppl 1996;75:S89-90.

[12] Usuda J, Tsutsui H, Honda H, Ichinose S, Ishizumi T, Hirata T, et al. Photodynamic therapy for lung cancers based on novel photodynamic diagnosis using talaporfin sodium (NPe6) and autofluorescence bronchoscopy. Lung Cancer 2007;58:317-23.

[13] Kimura T, Takatsuki S, Miyoshi S, Fukumoto K, Takahashi M, Ogawa E, et al. Nonthermal cardiac catheter ablation using photodynamic therapy. Circ: Arrythmia Electrophysiol 2013;6:1025-31.

[14] Ito A, Miyoshi S, Kimura T, Takatsuki S, Fukumoto K, Fukuda K, et al. Myocardial electrical conduction block induced by photosensitization reaction in exposed porcine hearts in vivo. Lasers Surg Med 2011;43:984-90.

[15] Spikes JD, Bommer JC. Photobleaching of mono-1-aspartyl chlorin e6 (NPe6): a candidate sensitizer for the photodynamic therapy of tumors. Photochem Photobiol 1993;58:346-50.

[16] Kessel D. Pharmacokinetics of N-aspartyl chlorin e6 in cancer patients. J Photochem Photobiol B 1997;39:81-3.

[17] Akimoto J, Haraoka J, Aizawa K. Preliminary clinical report on safety and efficacy of photodynamic therapy using talaporfin sodium for malignant gliomas. Photodiagn Photodyn Therapy 2012;9:91-9.

[18] Bromley E, Briggs B, Keltner L, Wang SS. Characterization of cutaneous photosensitivity in healthy volunteers receiving talaporfin sodium. Photodermatol Photoimmunol Photomed 2011;27:85-9.

[19] Ferrario A, Kessel D, Gomer CJ. Metabolic properties and photosensitizing responsiveness of mono-L-aspartyl chlorin e6 in a mouse tumor model. Cancer Res 1992;52:2890-3.

[20] Canpolat M, Mourant JR. Monitoring photosensitizer concentration by use of a fiber-optic probe with a small source-detector separation. Appl Opt 2000;34:6508-14.

[21] Weersink RA, Hayward JE, Diamond KR, Patterson MS. Accuracy of noninvasive in vivo measurements of photosensitizer uptake based on a diffusion model of reflectance spectroscopy. Photochem Photobiol 1997;66:326-35.

[22] Anderson RR, Parrish JA. The optics of human skin. J Investig Dermatol 1981;77:13-9.

[23] Cheong WF, Prahl SA, Welch AJ. A review of the optical properties of biological tissues. IEEE J Quantum Electron 1990;26:2166-85.

[24] Bashkatov AN, Genina EA, Kochubey VI, Tuchin VV. Optical properties of human skin, subcutaneous and mucous tissues in the 
wavelength range from 400 to $2000 \mathrm{~nm}$. J Phys D: Appl Phys 2005; 38:2543-55.

[25] Chan EK, Sorg B, Protsenko D, O’Neil M, Motamedi M, Welc AJ. Effects of compression on soft tissue optical properties. IEEE J Quantum Electron 1996;2:943-50.

[26] Mitra S, Foster TH. In vivo confocal fluorescence imaging of the intratumor distribution of the photosensitizer Mono-LAspartylchlorin-e6. Neoplasia 2008;10:429-38.

[27] Yamaoka K, Nakagawa T, Uno T. Statistical moments in pharmacokinetics. J Pharmacokinet Biopharm 1978;6: 547-58.
[28] Tsoukas MM, Lin GC, Lee MS, Anderson RR, Kollias N. Predictive dosimetry for threshold phototoxicity in photodynamic therapy on normal skin: red wavelengths produce more extensive damage than blue at equal threshold doses. J Investig Dermatol 1997; 108:501-5.

[29] Fingar VH, Henderson BW. Drug and light dose dependence of photodynamic therapy: a study of tumor and normal tissue response. Photochem Photobiol 1987;46:837-47.

[30] Kuppenheim HF, Heer RR. Spectral reflectance of white and negro skin between 440 and $1000 \mathrm{~m} \mu$. J Appl Physiol 1952;4:800-6. 